\title{
Modeling the electrical properties of three-dimensional printed meshes with the theory of resistor lattices
}

Citation for published version (APA):

Melnikov, A. V., Shuba, M., \& Lambin, P. (2018). Modeling the electrical properties of three-dimensional printed meshes with the theory of resistor lattices. Physical Review E, 97(4), [043307]. https://doi.org/10.1103/PhysRevE.97.043307

Document status and date:

Published: 17/04/2018

DOI:

10.1103/PhysRevE.97.043307

Document Version:

Publisher's PDF, also known as Version of record

Document license:

Taverne

\section{Please check the document version of this publication:}

- A submitted manuscript is the version of the article upon submission and before peer-review. There can be important differences between the submitted version and the official published version of record.

People interested in the research are advised to contact the author for the final version of the publication, or visit the DOI to the publisher's website.

- The final author version and the galley proof are versions of the publication after peer review.

- The final published version features the final layout of the paper including the volume, issue and page numbers.

Link to publication

\footnotetext{
General rights rights.

- You may freely distribute the URL identifying the publication in the public portal. please follow below link for the End User Agreement:

www.umlib.nl/taverne-license

Take down policy

If you believe that this document breaches copyright please contact us at:

repository@maastrichtuniversity.nl

providing details and we will investigate your claim.
}

Copyright and moral rights for the publications made accessible in the public portal are retained by the authors and/or other copyright owners and it is a condition of accessing publications that users recognise and abide by the legal requirements associated with these

- Users may download and print one copy of any publication from the public portal for the purpose of private study or research.

- You may not further distribute the material or use it for any profit-making activity or commercial gain

If the publication is distributed under the terms of Article $25 \mathrm{fa}$ of the Dutch Copyright Act, indicated by the "Taverne" license above, 


\title{
Modeling the electrical properties of three-dimensional printed meshes with the theory of resistor lattices
}

\author{
Alexander V. Melnikov, ${ }^{1}$ Mikhail Shuba, ${ }^{1,2}$ and Philippe Lambin ${ }^{3}$ \\ ${ }^{1}$ Institute for Nuclear Problems, Belarus State University, Bobruiskaya 11, 220050 Minsk, Belarus \\ ${ }^{2}$ Tomsk State University, 36 Lenin Avenue, Tomsk 634050, Russia \\ ${ }^{3}$ Physics Department, Université de Namur, 61 Rue de Bruxelles, B-5000 Namur, Belgium
}

(Received 31 December 2017; revised manuscript received 2 April 2018; published 17 April 2018)

\begin{abstract}
The electrical properties of conducting meshes are investigated numerically by solving the related Kirchhoff equations with the Lanczos algorithm. The method is directly inspired by the recursion technique widely used to study the electronic and vibrational spectra of solids. The method is demonstrated to be very efficient and fast when applied to resistor networks. It is used to calculate equivalent resistances between arbitrary pairs of nodes in simple resistive lattices. When the resistance fluctuates statistically from bond to bond, the method makes it possible to evaluate the fluctuations of the electrical properties of the network. It is also employed to assign an effective bulk resistivity to a discrete conducting three-dimensional mesh.
\end{abstract}

DOI: 10.1103/PhysRevE.97.043307

\section{INTRODUCTION}

Three-dimensional (3D) printers make it possible to design unprecedented structures with a geometry specially designed to reach specific properties [1]. For example, the making of lightweight periodic cellular architectures by $3 \mathrm{D}$ printing has been demonstrated [2]. Polymer filled with graphene nanoplatelets [3], multiwall carbon nanotubes [4], or other forms of $\mathrm{sp}^{2}$ nanocarbons may be used for 3D printing. Dispersing a few atomic percentage of these nanomaterials suffices to reach a good electrical conductivity of the host material $[5,6]$. Depending on the polymer matrix and the filler, these composites may have different mechanical, electrical, and thermal properties [5-7]. By feeding a 3D printer with polymerconducting composites, light porous conducting media can be fabricated $[2,4,8]$. The final product may be a periodic network of interconnected rods with millimeter-size length. Figure 1 is an illustrative example. Cellular conductive carbon foams with a structure similar to the considered 3D printed networks have significant electromagnetic shielding efficiency [9]. Thus, we assume that 3D printed meshes also may be effective in electromagnetic interference-shielding applications [10]. Other applications such as electrostatic discharge protection [11] can be foreseen for these artificial periodic networks.

Theoretical modeling of conductive properties of periodic networks is an important issue for applications. It can be used to choose materials and geometrical parameters of the lattice cells yielding the largest possible effective conductance at a given mass density. It makes it possible to investigate theoretically the electrical properties of mesh structures which are expected to have the best mechanical stiffness. Also, taking into account the fluctuations of the electrical resistance of the lattice edges is important, because fluctuations of the printing parameters and composite composition cannot be avoided in practice.

The conductive properties of two-dimensional (2D) or 3D meshes resort to the so-called theory of resistor networks that dates back to the 1970s [12-16]. These networks consist of resistors interconnected at points called nodes. This theory is applicable here due to the similarity between printed meshes and resistor networks: the rods correspond to the resistors in a resistor network, and the crossing points between the rods correspond to the nodes.

The theory of resistor networks is a simple formulation of Ohm's and Kirchhoff's laws that leads to a set of linear equations to be solved for the node potentials. Each equation in the system may be expressed in terms of the so-called lattice Laplacian [15]. For square and simple-cubic lattices, the lattice Laplacian corresponds to second-order finite-difference representations of the Laplace operator. Mathematically, the problem may be seen as a discretized Poisson's equation [17].

There are different interesting tasks related to the theory of resistor networks: (1) the solution of the discrete Poisson-like equation in the presence of current sources can be applied, for example, for the calculation of the equivalent resistance between two arbitrary nodes of the lattice $[15,16]$; (2) the resolution of the problem with appropriate boundary conditions for finite-size systems (for instance, obtaining the electrical potential distribution in the network when the electrical potential is set at predefined values at some boundaries of the model can be used to define an effective conductivity of the lattice) $[12,14]$; and (3) a combination of the previous tasks [17].

The equivalent resistance of a conductive mesh seen from a given pair of nodes allows one to characterize the system from the electrical point of view. Analytical methods can be used to calculate the equivalent resistance between two arbitrary nodes in a perfect resistor network. For simple lattices, this particular task can be solved in terms of lattice Green's functions [15]. Analytical formulas are available for triangular, honeycomb, square, rectangular, diamond, simplecubic, body-centered cubic, face-centered cubic, and hypercubic (simple cubic in $N$ dimensions) lattices [18-22]. Also, Green's function approach has been applied to uniform tiling of space with electrical resistors [23]. The lattice Green's function formalism can be developed to address several types of defects, 
(a)

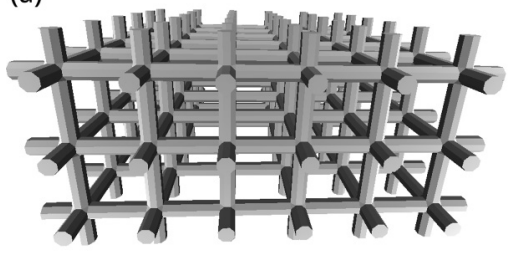

(b)

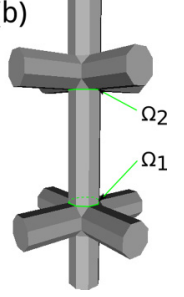

FIG. 1. (a) Periodic network of rods (octagonal prisms) meeting at nodes located on a simple cubic lattice. (b) Detail of a rod with its two ending sections $\Omega_{1}$ and $\Omega_{2}$.

including a broken resistor and an extra resistor between two initially nonconnected nodes [24,25]. The way defects can be dealt with is an iterative process that can be extended to the simultaneous presence of several defects. However, the number of defects is limited by the computational complexity of the iterative process. By comparison, numerical methods have no limitations in the concentration of defects, as long as the network remains of finite size. An accurate solution of the problem can be obtained by numerical methods for networks containing up to several million resistors per processor core. Nowadays, it is possible to solve linear problems with one trillion unknowns on supercomputers [26].

In this work, Lanczos's algorithm has been used to solve the discretized Poisson-like equation. This algorithm, also known in physics as the recursion method [27], can be applied to this problem [28], because the Laplacian matrix, being sparse and symmetric, can be efficiently reduced to a tridiagonal form. The tridiagonal form is then used to express any diagonal element of the lattice Green's function in a continued fraction [29]. Being an order of $N$ for a given fixed number of recursion steps, with $N$ the number of nodes, this method may reach high performance and requires little memory storage. Moreover, it can be corrected to extrapolate the results to infinite networks. This method has been used in the present work to calculate the equivalent resistance between two nodes in different kinds of infinite lattices, with and without defects. Results for some simple infinite lattices are compared with available analytical results.

The equivalent resistance that can be calculated in a resistor network is what would be obtained experimentally with a two-probe measurement technique. In a continuous medium, the relevant quantity is the resistivity, which is best measured by a four-probe method. Calculations mimicking a four-probe experiment have also been carried out for 3D resistor lattices to get an effective bulk resistivity. This quantity is an important ingredient if one wants to compare the transport properties of a conducting mesh with that of a continuous medium. The effective resistivity can be further used to calculate the skin depth and electromagnetic interference-shielding effectiveness of the 3D discrete network.

The paper is organized as follows. After a brief description of the equivalence between 3D meshes and resistor networks, basic electrokinetic equations are derived in Sec. II. Then the recursion algorithm used to solve these equations is presented. Two ways of improving the accuracy of the numerical results for infinite networks are proposed and discussed. In Sec. III an effective conductivity is assigned to a discrete resistor network, the aim being to mimic a conducting mesh by a continuous medium. Both global and local point of views are explored. Conclusions are drawn in Sec. IV. Some computational properties of the method are described in the Appendix.

\section{THEORY OF RESISTIVE LATTICES}

We assume that a 3D printed mesh, such as the one illustrated in Fig. 1(a), is a periodic lattice of rods (called edges or bonds) of length $L$, cross section $S$, and conductivity $\sigma_{0}$. The edges may have different lengths depending on the lattice geometry. In a rectangular lattice, for instance, edges in one direction are shorter than the edges in the perpendicular direction. Each edge in the network may be characterized by its resistance $R=L /\left(S \sigma_{0}\right)$. The edge resistance may vary due to fluctuations of $L, S$, and $\sigma_{0}$ of the rods produced by the 3D printer. In some situations, a few bonds are broken. How to deal with some randomness of the resistors is described in Sec. II C.

Each node of a resistive mesh dispatches the current it receives from an external source among the rods attached to it. According to Kirchhoff's law, the sum of the currents flowing to a node is zero. Whatever the exact distribution of the current density $\vec{j}$ across a rod, like the octagonal prism shown in Fig. 1(b), the potential drop between its two ending faces $\Omega_{1}$ and $\Omega_{2}$ is related to the total current $I$ flowing through it by Ohm's law $\Delta V=R I$. Here $\Delta V=(1 / S) \int_{\Omega_{1}} V d S-$ $(1 / S) \int_{\Omega_{2}} V d S$, with $S$ the common area of $\Omega_{1}$ and $\Omega_{2}$. This result is obtained by integrating $-\partial V / \partial z=j_{z} / \sigma_{0}$ in the volume of the rod, where $z$ is a local axial coordinate. The bases $\Omega_{1}$ and $\Omega_{2}$ of the rod are two faces of the nodes interconnected by the rod [see Fig. 1(b)]. We shall make the approximation that each node is an equipotential volume that can therefore be characterized by a single potential. Then $\Delta V$ becomes the potential difference between the nodes interconnected by the rod. The equipotential hypothesis is valid if the nodes are made of a highly conducting medium or if their linear dimensions are small compared to the length of the rods. The first case supposes that nodes and rods of the mesh are made of two different materials, which is indeed possible to achieve with a $3 \mathrm{D}$ printer. The second case is more realistic, especially if the nodes are truncated polyhedra, like the cubes truncated at their corners by height triangular faces in Fig. 1(b), that occupy the smallest possible volume.

Under the hypothesis that a unique potential can be attributed to each node, a resistive mesh can be approximated by a resistor network. Let us therefore apply Ohm's and Kirchhoff's laws to the nodes of the network:

$$
\sum_{j \in Z(i)} g_{i j}\left(V_{j}-V_{i}\right)+I_{i}=0
$$

where $Z(i)$ is the set of nodes $j$ connected to the $i$ th node through resistors, $g_{i j}$ is the conductance of the resistors connecting node $i$ to node $j, V_{i}$, and $V_{j}$ are the electric potentials of nodes $i$ and $j$, respectively, and $I_{i}$ is the external current injected into node $i$.

The set of equations (1) may be rewritten in a compact form by introducing the Laplacian matrix $\mathbf{L}$ :

$$
\sum_{j \in\{i, Z(i)\}} L_{i j} V_{j}=-I_{i}, \quad L_{i j}=g_{i j} j \neq i, \quad L_{i i}=-\sum_{j \in Z(i)} g_{i j},
$$


and transformed in a matrix equation:

$$
\mathbf{L V}=-\mathbf{I},
$$

where $\mathbf{I}$ is the vector of external current sources connected to the resistor network, and $\mathbf{V}$ is the vector of the unknown node potentials.

The Laplacian matrix in (3) is singular. To solve the system (3) we transform it as

$$
(y \mathbb{1}-\mathbf{L}) \mathbf{V}=\mathbf{I},
$$

where $\mathbb{1}$ is the identity matrix and $y$ is a real positive conductance. All the nodes are thereby grounded to an absolute reference of potential set to zero. We shall take the limit $y \rightarrow 0+$ afterward. The set of equations (4) can be solved numerically for any finite-size lattice by application of some high-performance direct methods, like block methods [30], and iterative methods, like SOR [30] or conjugate-gradient [31] methods. The formulation of the problem (4) is general and allows us to find the node potentials generated by a given distribution of external current sources. In the absence of current sources, some node potentials can receive predefined values (Dirichlet boundary conditions), and all the terms involving imposed potentials are transferred to the right-hand side, where they play the same role as the vector $I$ in Eq. (4) [17]. This latter kind of problem with Dirichlet boundary conditions is involved in numerical calculation of the equivalent bulk resistivity for 3D printed meshes; see Sec. III.

One way to solve Eq. (4) is via the resolvent matrix:

$$
\mathbf{r}(y)=(y \mathbb{1}-\mathbf{L})^{-1} .
$$

The node potentials may thus be expressed from Eqs. (4) and (5) as

$$
\mathbf{V}=\lim _{y \rightarrow 0+} \mathbf{r}(y) \mathbf{I} .
$$

There are two particular tasks for which the vector $\mathbf{I}$ consists of a current source and a current sink: $I_{k}=I_{0}\left(\delta_{k, i}-\delta_{k, j}\right)$; namely, the current $I_{0}$ enters the network at node $i$ and leaves it at node $j$. The first task involves four nodes if we assume that the voltage nodes $m$ and $n$ between which $\Delta V=V_{m}-V_{n}$ is computed are distinct from the current nodes $i$ and $j$. This setup is analogous to the four-probe method widely used for measuring the resistivity of bulk materials [32]. The second task represents a two-probe setup yielding the equivalent resistance $R(i, j)$ between two arbitrary nodes $i$ and $j$ of the resistor network. Here the current and voltage electrodes coincide.

The potential difference in the four-probe setup is

$$
V_{m}-V_{n}=\left(r_{m i}-r_{m j}-r_{n i}+r_{n j}\right) I_{0},
$$

where $r_{m i}$ represents the limit of the $(m, i)$ element of the resolvent matrix $r(y)$ for a vanishingly small positive $y$. The determination of $R(i, j)$ follows from the four-probe case by setting $m=i$ and $n=j$ :

$$
R(i, j)=\frac{V_{i}-V_{j}}{I_{0}}=\left(r_{i i}-r_{i j}-r_{j i}+r_{j j}\right) .
$$

$R(i, j)$ can been obtained numerically for ideal simple lattices of finite size after the eigenvalues and eigenvectors of the Laplacian matrix have been computed [16]. For ideal infinite resistor networks, the resolvent matrix in Eq. (5) is the lattice Green's function $G$. When analytical expressions of $G$ exist, $R(i, j)$ can be calculated with high accuracy. The lattice Green's function is available for square, rectangular, triangular, simple cubic, and some other lattices [18-23]. The perturbation of the lattice Green's function by defects in ideal networks has been considered [24,25]. It allows one to obtain $R(i, j)$ in infinite networks containing a small number of defective resistors.

Analytical methods based on Green's functions are restricted to simple lattices and limited by the number of defective resistors in the network. Numerical methods for the basic system of equations (4) are free from these limitations but can be applied to finite-size systems only. Compared to an infinite lattice, numerical results obtained on a finite network deviate from the values given by direct analytical methods based on Eq. (6) and Green's functions. The error may be expressed as

$$
\delta R(i, j)=\left|R(i, j)_{n}-R(i, j)_{a}\right|,
$$

where $R(i, j)_{a}$ is the equivalent resistance between two nodes calculated by direct analytical method, and $R(i, j)_{n}$ is the same calculated by numerical methods.

The error $\delta R(i, j)$ is due to the finite size of the system and the concomitant neglect of paths of the current across resistors that are outside the boundaries of the network. If the linear size of the system increases to $\infty$, then assuming an error-free algorithm, one obtains $R(i, j)_{n} \rightarrow R(i, j)_{a}$ and $\delta R(i, j) \rightarrow 0$. In practice, the size of the system is limited by the amount of computational operations and memory storage. However, there is another way to increase the accuracy of the calculation by the inclusion of some corrections in the calculation procedure; see Sec. II B.

\section{A. Recursion algorithm for numerical calculation of the equivalent resistance between two nodes of a resistor network}

For the calculation of, e.g., the equivalent resistance $R(i, j)$ between two nodes of an infinite network, we use the recursion method initially designed for a tight-binding Hamiltonian in solid-state physics [29]. To sketch how the method works, we first rewrite Eq. (8) in Dirac notation:

$$
R(i, j)=2 \lim _{y \rightarrow 0+}\left\langle u_{i j}|\mathbf{r}(y)| u_{i j}\right\rangle \quad \text { with }\left|u_{i j}\right\rangle=\frac{|i\rangle-|j\rangle}{\sqrt{2}} .
$$

Here $\left|u_{i j}\right\rangle$ is a normalized vector in the space sustained by the nodes of the network, which has zero elements everywhere except at the nodes $i$ and $j$, where it takes the values $+1 / \sqrt{2}$ and $-1 / \sqrt{2}$, respectively. It is worth mentioning here that, unlike in Ref. [28], the equivalent resistance is obtained in one step as a diagonal element of the resolvent matrix [Eq. (10)]. There is no need to calculate separately diagonal elements $r_{i i}$ and off-diagonal ones $r_{i j}$, as Eq. (8) would suggest, which improves the precision and saves computing time.

Starting from the state $\mid 0\}=\left|u_{i j}\right\rangle$, the recursion method generates recursively a set of normalized states through the relation

$$
\left.\left.b_{m+1}|m+1\rangle=\left(\mathbf{L}+a_{m+1} \mathbb{1}\right) \mid m\right\}-b_{m} \mid m-1\right\},
$$




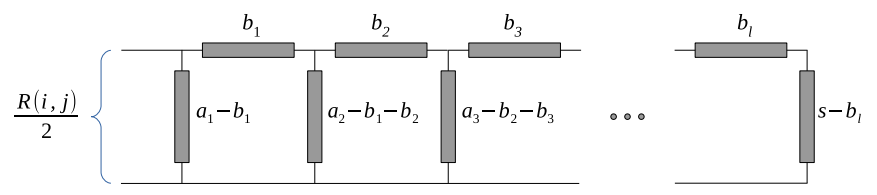

FIG. 2. Physical interpretation of the continued fraction [Eq. (12)] in the form of a transmission line. Each gray-shaded box represents a resistor whose conductance is indicated along it.

where the so-called recursion coefficients $a_{m}$ and $b_{m}$ are defined at each step of the recurrence in such a way that the state $\mid m\}$ be orthogonal to all the states obtained that far. In the basis defined by these states, the Laplacian matrix is transformed in a tridiagonal form, with coefficient $-a_{m}$ as diagonal element in the $m$ th row and coefficients $b_{m-1}$ and $b_{m}$ on both sides of $a_{m}$ in the same row.

After $l$ steps, $l$ pairs of recursion coefficients are obtained from Eq. (11). Using the definition of the resolvent matrix and the recurrence relation (11), one obtains

$$
\begin{aligned}
\{0|\mathbf{r}(y)| 0\} & =\frac{1}{y+a_{1}-b_{1} \frac{\{0|\mathbf{r}(y)| 1\}}{\{0|\mathbf{r}(y)| 0\}}}, \\
\frac{\{0|\mathbf{r}(y)| m\}}{\{0|\mathbf{r}(y)| m-1\}} & =\frac{b_{m}}{y+a_{m+1}-b_{m+1} \frac{\{0|\mathbf{r}(y)| m+1\}}{\{0|\mathbf{r}(y)| m\}}} \\
m & =1,2, \ldots
\end{aligned}
$$

Combining these two relations together with Eq. (10) yields a continued-fraction expansion for the equivalent resistance:

$$
R(i, j)=\frac{2}{a_{1}-\frac{b_{1}^{2}}{a_{2}-\cdots \frac{b_{l-1}^{2}}{a_{l}-\frac{b_{l}^{2}}{s}}},}
$$

where $s$ stands for all the terms that are not calculated when stopping the recurrence at step $l$. The $l$ th approximant of the continued fraction corresponds to the limit $s \rightarrow \infty$. The set of approximants may converge slowly with increasing $l$. In practice, however, the convergence can be accelerated by using a suitable, finite termination $s$. Interpreting $a_{1}, b_{1}, a_{2} \ldots b_{l}$, and $s$ as conductances, Eq. (12) can be viewed as the input resistance of the transmission line illustrated in Fig. 2. Physically, the load conductance $s-b_{l}$ should be positive. According to this criterion, a value of $s$ greater than $b_{l}$ must be used to terminate the continued fraction (12). Hereafter, we shall refer to the value $s=b_{l}$ as a "minimal termination parameter."

For an infinite lattice, there is a relation between the size of the cluster used for the calculations and the number $l$ of continued-fraction levels one may consider to calculate the equivalent resistance $R(i, j)$ between nodes $i$ and $j$. In the case of periodic boundary conditions, artificial periodicity starts playing a role if the shortest percolation path connecting site $i$ to the closest translational duplicate of site $j$ comprises less than $2 l$ bonds. For the case of free boundary conditions, the surface starts to influence the results if there exists a path composed of less then $2 l$ bonds going from $i$ to $j$ while passing
TABLE I. The computational complexity of the recursion algorithm for the calculation of the equivalent resistance between two nodes of a 2D square lattice and a 3D simple-cubic lattice is compared with some other algorithms that can be used for solving Poisson's equation in two and three dimensions.

\begin{tabular}{lcc}
\hline \hline & \multicolumn{2}{c}{ Computational complexity } \\
\cline { 2 - 3 } Algorithm & $2 \mathrm{D}$ & $3 \mathrm{D}$ \\
\hline Recursion (this work) & $O\left(N^{3 / 2}\right)$ & $O\left(N^{4 / 3}\right)$ \\
Block [30] & $O\left(N^{3 / 2}\right)$ & \\
Star-mesh transformation [33] & $O\left(N^{3 / 2}\right)$ & \\
Conjugate gradient [31] & $O\left(N^{3 / 2}\right)$ & $O\left(N^{4 / 3}\right)$ \\
SOR [30,34] & $O\left(N^{3 / 2} \cdot \log N\right)$ & $O\left(N^{4 / 3} \cdot \log N\right)$ \\
Jacobi and Gauss-Seidel [34] & $O\left(N^{2} \cdot \log N\right)$ & $O\left(N^{5 / 3} \cdot \log N\right)$ \\
\hline \hline
\end{tabular}

through a surface node. This rule is illustrated for small values of $l$ in Fig. 5 below: the computed value of the equivalent resistance between two first-neighbor nodes does not change by increasing the linear size of the cluster as soon as the latter exceeds $2 l$.

Let us compare the efficiency of the recursion algorithm and other well-known algorithms that can be applied to solve 2D and 3D discrete Poisson's equation. In two dimensions (resp. three dimensions), the discrete Laplace operator obtained by five-point (resp. seven-point) stencil finite-difference methods has the same form as the Laplacian matrix given by Eq. (2) for a square (resp. simple-cubic) resistor lattice. Solving Eq. (4) for these particular resistor lattices is equivalent to solving a discrete Poisson equation. Thus we can compare the computational complexity of the recursion algorithm applied to the calculation of the equivalent resistance $R(i, j)$ between two nodes of square and simple cubic networks with the computational complexity of $2 \mathrm{D}$ and $3 \mathrm{D}$ discrete Poisson equation solvers, respectively. We consider square networks with size (number of nodes) $N=n \cdot n$ and simple-cubic networks with size $N=n \cdot n \cdot n$.

As shown in the Appendix, the computational complexity of the recursion method for a three-dimensional lattice is $O\left(N^{4 / 3}\right)$. In two dimensions, the computational complexity becomes $O\left(N^{3 / 2}\right)$ when the number of continued-fraction levels $l$ is optimized to the linear size of the network, which scales with $N$ like $n=N^{1 / d}$, with $d$ the dimension of space. As demonstrated by Table I for both $2 \mathrm{D}$ and $3 \mathrm{D}$ problems, the recursion algorithm can compete with direct block methods [30], star-mesh transformation [33], conjugate gradient [31], and the iterative successive over-relaxation (SOR) method [30,34]. Moreover, the recursion method should run faster than iterative Jacobi and Gauss-Seidel algorithms [34] according to their respective computational complexity. In addition to its relatively high speed, the recursion algorithm can be corrected to improve the accuracy of the results in the case of infinite lattices, as we now demonstrate.

\section{B. Improvement of accuracy for infinite resistor networks}

Here we present two ways of improving the accuracy of the recursion method applied to infinite resistor networks while necessarily dealing with a finite system. The first way 
(a)

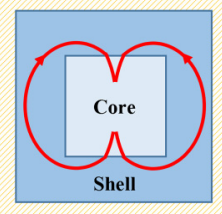

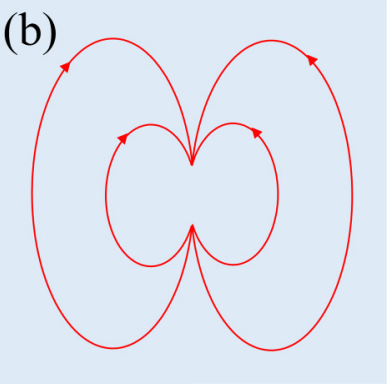

FIG. 3. Principles of the core-shell model. In (a), the conductivity of the boundary shell is higher than that of the core region allowing keeping within the finite-size region as many current lines as in the case of the infinite medium shown in (b). The core region in (a) has the same conductivity as the infinite medium shown in (b).

is to adjust the continued-fraction termination parameter $s$ [Eq. (12)] in such a way that $R(i, j)_{n}$ between two nearestneighbor nodes $i$ and $j$ reproduces the analytical result $R(i, j)_{a}$, when available [35]. The parameter $s$ so obtained is used for all pairs $i, j$ of nodes. It is called the optimized termination (OT) parameter in the following. Its value depends on the lattice and the bond resistance, on the size of the cluster, and on the number $l$ of continued-fraction levels. The second way consists in decreasing the resistance of the resistors in some boundary region of finite-size medium (core-shell model); see Fig. 3. The thickness of a boundary shell (BS) is taken to be equal to a number of resistors which connect the core region with the outer part of the network. The higher conductivity of the boundary shell provides a larger current in it, which permits one to replace the current in the outer infinite network by the current in the shell. The resistance in the shell is chosen in such a way that the computed equivalent resistance between two nearest-neighbor nodes possesses the exact value. For perfect resistor networks in which each node is connected to $Z$ equivalent bonds with resistance $R_{0}$, the equivalent resistance between a pair of first-neighbor nodes is $2 R_{0} / Z$. The value of $Z$ is referred to as the coordination number of the lattice.

To illustrate these concepts, let us consider an ideal square lattice with bond resistance $R_{0}$ set to 1 . The equivalent resistance $R(i, j)$ is calculated on a $100 \times 100$ network between a node $j$ with coordinates $(m, n)$ and the node $i$ located at the geometrical center of the network where it receives the coordinates $(0,0)$. Different sets of coordinates $(n, m)$ of the node $j$ are considered. For comparison, the equivalent resistance has been computed analytically using Green's function. The accuracy of the recursion method is measured with the absolute error $\delta R(i, j)$ [Eq. (9)]. Three types of numerical calculations are presented: (1) calculations with $l=60$ continued-fraction levels using the minimum value of termination parameter, $s=b_{l}$; (2) calculations still with $l=60$ and the termination parameter $s=2.01426$ adjusted to get the correct value of the first-neighbor equivalent resistance (+OT); (3) calculations with the core-shell model, now with $l=200$, the boundary shell having a thickness of 25 resistors and a bond resistance $R_{1}=$ 0.56351 (+BS). In the latter calculations, $s=b_{l}$ is used, as in calculations (1).

As Table II indicates, the recursion algorithm with the minimum termination parameter allows one to obtain $R(i, j)$

TABLE II. Analytical results for the equivalent resistance between two nodes $R(i, j)_{a}$ for the ideal square lattice and absolute error $\delta R(i, j)$ of numerical calculations based on the recursion algorithm (see text).

\begin{tabular}{ccccc}
\hline \hline$(n, m)$ & $R(i, j)_{a}$ & $\delta R(i, j)$ & $\delta R(i, j)+\mathrm{OT}$ & $\delta R(i, j)+\mathrm{BS}$ \\
\hline$(1,0)$ & 0.50000000 & 0.00002400 & 0.00000001 & 0.00000010 \\
$(2,0)$ & 0.72676045 & 0.00011439 & 0.00001083 & 0.00000026 \\
$(4,0)$ & 0.95398729 & 0.00044407 & 0.00003788 & 0.00000317 \\
$(1,1)$ & 0.63661977 & 0.00005752 & 0.00001311 & 0.00000025 \\
$(2,2)$ & 0.84882636 & 0.00022619 & 0.00002047 & 0.00000062 \\
$(4,4)$ & 1.06709599 & 0.00086582 & 0.00006492 & 0.00000875 \\
\hline \hline
\end{tabular}

with an accuracy of three decimal figures. The correction based on the adjusted termination of the continued fraction improves the accuracy by at least a factor of 10 . It is worth noting that accuracy has improved for all the equivalent resistances considered. The core-shell model requires more continuedfraction levels in order to better probe the shell boundary zone. Meanwhile, it leads to improving the accuracy by at least a factor of 100 for all configurations of nodes.

Let us now consider a square network of size $100 \times 100$ with a single broken bond between two nearest-neighbor nodes. Analytical calculation of $R(i, j)_{a}$ is provided using perturbation of the lattice Green's function. Numerical methods for the calculation of $R(i, j)_{n}$ are the same as for the ideal square network. In particular, the same termination parameter $s=2.01426$ is used for the (+OT) improved calculations.

The same level of accuracy as for the perfect network is obtained when using the recursion algorithm with either (+OT) or $(+\mathrm{BS})$ corrections. By contrast, $\delta R(i, j)$ with the minimal continued-fraction termination (the third column of Table III) increases by a factor varying between five and 10. One may conclude that the recursion method remains efficient for a network containing a local defect, if one applies the same corrections as for the ideal network.

To illustrate the accuracy of numerical calculations of $R(i, j)_{n}$ for 3D infinite networks, we consider an ideal simple cubic lattice. Direct analytical results have been calculated with Green's functions. Numerical results have been obtained with the recursion algorithm with and without adjusted termination.

TABLE III. Analytical results for the equivalent resistance between two nodes $R(i, j)_{a}$ for a square lattice with a single broken bond between nodes $(0,0)$ and $(1,0)$. Absolute error $\delta R(i, j)$ of numerical calculations is based on the recursion algorithm.

\begin{tabular}{ccccc}
\hline \hline$(n, m)$ & $R(i, j)_{a}$ & $\delta R(i, j)$ & $\delta R(i, j)+\mathrm{OT}$ & $\delta R(i, j)+\mathrm{BS}$ \\
\hline$(1,0)$ & 1.00000000 & 0.00035614 & 0.00000005 & 0.00000039 \\
$(2,0)$ & 0.99085083 & 0.00065079 & 0.00002707 & 0.00000058 \\
$(4,0)$ & 1.13006365 & 0.00178588 & 0.00007674 & 0.00000274 \\
$(0,1)$ & 0.56602259 & 0.00010111 & 0.00000259 & 0.00000010 \\
$(0,2)$ & 0.82960106 & 0.00036826 & 0.00001378 & 0.00000022 \\
$(0,3)$ & 1.07364385 & 0.00137990 & 0.00005134 & 0.00000322 \\
$(1,1)$ & 0.83926213 & 0.00032515 & 0.00002758 & 0.00000045 \\
$(2,2)$ & 1.01447645 & 0.00091827 & 0.00002940 & 0.00000105 \\
$(4,4)$ & 1.21246892 & 0.00302011 & 0.00008516 & 0.00000724 \\
\hline \hline
\end{tabular}


TABLE IV. Analytical results for the equivalent resistance between two nodes $R(i, j)_{a}$ in the ideal simple cubic lattice. Absolute error $\delta R(i, j)$ of numerical calculations is based on the recursion algorithm with the minimum termination parameter $s=b_{l}$ (third column) and with an adjusted value of $s$ (fourth column).

\begin{tabular}{lccc}
\hline \hline$(n, m, l)$ & $R(i, j)_{a}$ & $\delta R(i, j)$ & $\delta R(i, j)+\mathrm{OT}$ \\
\hline$(1,0,0)$ & 0.33333333 & 0.00002374 & 0.00000000 \\
$(2,0,0)$ & 0.41968339 & 0.00008600 & 0.00000306 \\
$(3,0,0)$ & 0.45037176 & 0.00017940 & 0.00000594 \\
$(1,1,0)$ & 0.39507915 & 0.00004352 & 0.00000250 \\
$(1,2,0)$ & 0.43359881 & 0.00010764 & 0.00000133 \\
$(2,2,0)$ & 0.44935167 & 0.00016347 & 0.00000719 \\
$(1,1,1)$ & 0.41830531 & 0.00006334 & 0.00000338 \\
$(2,2,2)$ & 0.46015929 & 0.00023483 & 0.00001176 \\
$(3,3,3)$ & 0.47502340 & 0.00042933 & 0.00004596 \\
$(1,2,3)$ & 0.46314670 & 0.00025823 & 0.00002058 \\
\hline \hline
\end{tabular}

The size of the network is $N=45 \times 45 \times 45$, and the resistance of each resistor is set to 1 . In both cases, calculations with $l=20$ continued-fraction levels are provided. By setting $s=3.14605$, the equivalent resistance between first-neighbor sites is correctly reproduced.

Results presented in Table IV demonstrate that the level of accuracy is similar as for the $2 \mathrm{D}$ square lattice, although the number of continued-fraction levels has been considerably reduced (20 against 60). Then the equivalent resistance $R(i, j)$ can be obtained with three significant figures with the minimal termination $s$ and with four significant figures when using the adjusted termination.

\section{Random distribution of the resistor values}

In principle, the disorder in a conducting mesh produced by a 3D printer can be described by some probabilistic distribution of the resistance $p(R)$ or conductance $p(g)$ of the bonds. It is possible to deal with such a disorder in the spirit of the mean field theory $[12,13]$. The theory is remarkably simple when the underlying lattice is infinite and the unit cell contains one node where $Z$ equivalent resistor bonds meet [12]. Then the real system can be replaced by an ideal, ordered one, with an effective bond conductance $g_{\text {eff }}$ :

$$
\int_{0}^{\infty} p(g) \frac{\left(g_{\text {eff }}-g\right)}{\left[g+(Z / 2-1) g_{\text {eff }}\right]} d g=0 .
$$

This equation states that, having selected one bond $i, j$ of the ideal system and letting the conductance of that bond vary according to the probability distribution $p(g)$, then the average value of the equivalent resistance $R(i, j)$ will be that of the perfect lattice, $2 /\left(Z g_{\text {eff }}\right)$. Once $g_{\text {eff }}$ has been calculated, one is back to the theory of ideal resistor networks. The equivalent resistance between any given pair of nodes $i$ and $j$ of the ideal network with bond conductance $g_{\text {eff }}$ has the meaning of a configurational averaged value of $R(i, j)$ for random resistor network [36]. It is worth noting that the accuracy of this approach increases with increasing coordination number $Z$ (see, e.g., Refs. [14,36]).

Determining the distribution function $p(g)$ for the bond resistors of a realistic 3D mesh is not an easy task. For simplicity, Gaussian distribution of the conductance of bond resistors can be assumed with mean value $g_{0}$ and standard deviation $\Delta g$. The Gaussian distribution is truncated on its negative tail in order to avoid negative values of $g$ : the tail on the negative side is pushed upward and replaced by a Dirac delta peak at the origin whose weight compensates for the weight of the truncated tail. This delta peak physically represents the probability that a rod in the $3 \mathrm{D}$ printed mesh be broken. $\mathrm{Nu}-$ merical simulations performed for the diamond lattice $(Z=4)$ are listed in Table $\mathrm{V}$. The equivalent resistance, computed from Eq. (13) with the truncated Gaussian distribution for $\Delta g / g_{0}=$ 0.1 , is $R_{\text {eff }}=1.005 g_{0}^{-1}$. The data in Table $\mathrm{V}$ demonstrate that the relative dispersion $\Delta R(i, j) /\langle R(i, j)\rangle$ of the equivalent resistance between any two nodes, calculated for a sample with 7000 different but equivalent configurations of the resistors, is less than the relative dispersion $\Delta g / g_{0}=0.1$ of the resistors themselves.

We have explored how the equivalent resistance fluctuates around its average value in a square lattice of resistors. The conductance of the resistors was chosen according to the truncated Gaussian distribution with $\Delta g / g_{0}=0.2$, and 1000 random configurations were generated. For each of them, the equivalent resistance $R(i, j)$ between the node $i$ located at the center $(0,0)$ of a $200 \times 200$ network and the node $j$ at coordinates $(d, 0)$ was calculated for $d=1,2, \ldots, 20$. The configurational averaged value $\langle R(i, j)\rangle$ is represented versus the distance $d$ between the nodes $i$ and $j$ in Fig. 4. The gray-scale maps visualize the fluctuations of $R(i, j)$, the horizontal blue bars represent the 5 th and 95 th percentiles. The 5 th-95th percentile interval keeps reasonably the same for all the distances $d>3$. The results of the perfect lattice with effective resistance

TABLE V. Configurational average of equivalent resistance in the diamond lattice with a truncated Gaussian distribution of the bond conductances (see text). The variance of the distribution is $\Delta g=0.1 g_{0}, g_{0}$ being the average value. Each resistance is given in units of $g_{0}^{-1}$. Calculations are performed on a $30 \times 30 \times 30$ supercell with the recursion algorithm. The number of continued fraction levels is 25 . We use the optimized termination parameter $s=2.0495 / R_{\text {eff }}$ (see Sec. II B) for the perfect diamond lattice with bond resistance $R_{\text {eff }}$.

\begin{tabular}{lccc}
\hline \hline Sites $i, j$ & $\langle R(i, j)\rangle$ & $\Delta R(i, j)=\sqrt{\left\langle[R(i, j)-\langle R(i, j)\rangle]^{2}\right\rangle}$ & Effective medium \\
\hline 1st neighbor & 0.502 & 0.026 & 0.5025 \\
2nd neighbor & 0.670 & 0.021 & 0.6700 \\
3rd neighbor & 0.715 & 0.019 & 0.7142 \\
4th neighbor & 0.747 & 0.019 & 0.7469 \\
5th neighbor & 0.749 & 0.019 & 0.7491 \\
6th neighbor & 0.778 & 0.019 & 0.7700 \\
$\infty$ & 0.898 & 0.026 & 0.9009 \\
\hline \hline
\end{tabular}




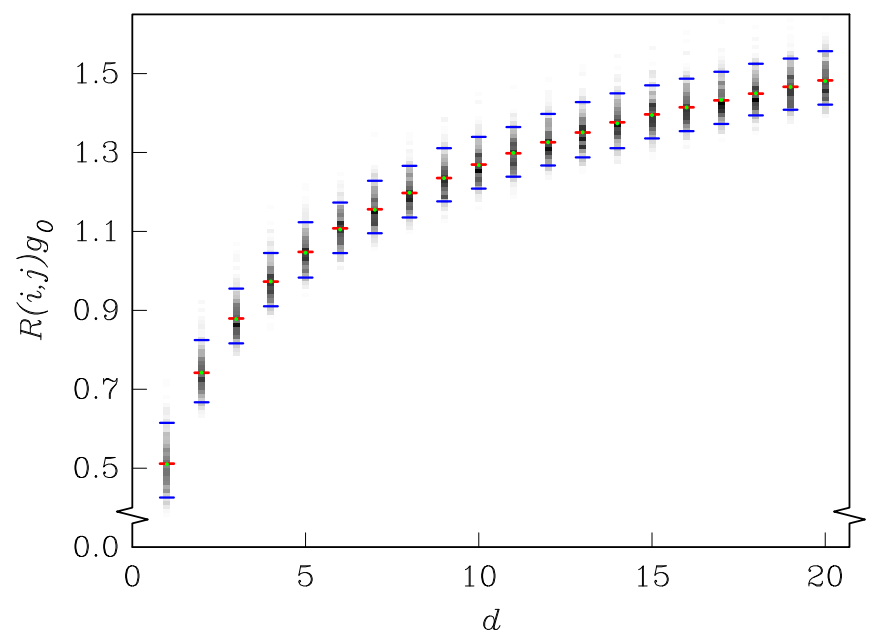

FIG. 4. Plot of the equivalent resistance $R(i, j)$ between two nodes $i$ and $j$ in a disordered square lattice of resistors. The nodes are along the $[1,0]$ direction and are separated by $d$ lattice parameters. The conductance of the resistors were taken randomly according to a truncated Gaussian distribution (see text) with average value $g_{0}$ and standard deviation $\Delta g=0.2 g_{0}$. The vertical gray patterns represent the fluctuations of equivalent resistances computed for 1000 independent configurations. The average values are shown by the horizontal red bars. The 5th and 95th percentiles of the fluctuations are shown by the horizontal blue bars. The green spots represent the results obtained on the same square lattice with a uniform resistance $R_{\text {eff }}$. In all cases, a $200 \times 200$ lattice was used, and the continued fraction (12) was truncated at its 75 th approximant.

$R_{\text {eff }}=1.021 g_{0}^{-1}$ are plotted in the same figure. They reproduce the configurational averaged data very well. The effective medium corresponds therefore to an efficient averaging of $R(i, j)$ and makes the theory useful to approximate a random resistor network by its equivalent ideal resistor network. The same effective-medium approach has also been applied to study the conductive properties of nanowire networks in two dimensions [37]. Here cross-connections between randomly dispersed nanorods form an irregular conducting net to which Eq. (13) does not apply directly. Previously the nanowire network has been mapped onto a regular square lattice where the bond resistances vary according to probability distributions of interwire junction resistances, intrawire resistances, and infinite-nonelectrically active wire segment-resistances. In spite of the regular network approximation used and complex distribution functions of the resistances in a nanowire network, the effective-medium theory accurately reproduces the numerical values of the equivalent resistance between two point electrodes similar to what is shown in Fig. 4 for a disordered square lattice with a truncated Gaussian distribution of resistances.

It is interesting that the fluctuations of equivalent resistance between two nodes of the square lattice considered in Fig. 4 do not vary significantly when $d$ exceeds 3 . A similar dependence of fluctuations of the equivalent resistance versus distance has been observed in the aforementioned modeling of 2D nanowire networks' topology [37]. In both cases, the random distribution of the resistance between connected nodes is the source of fluctuations. However, for the case of nanowire networks, an additional source of fluctuations exists: the geometry of the network changes from one arrangement of the nanowires to the other, whereas the geometry of our square lattice is kept the same for all resistor configurations. Despite this difference, a close similarity is observed in the way the fluctuations of the equivalent resistance depend on the distances between two nodes. In our case, we can imagine that the fluctuations come mainly from the finite number of resistance configurations inside a small region around each node $i$ and $j$. Outside these two small regions, the network offers so many different paths for the current that a configurational averaging is almost automatically performed. Then the fluctuations of $R(i, j)$ do not change with increasing the distance between the nodes above a given small value. The same trends are revealed by the data of Table $\mathrm{V}$ for the diamond structure: the standard deviation $\Delta R(i, j)$ keeps constant as soon as $j$ lies outside the third-coordination shell of $i$.

Irrespective of the type of distribution function, if $p(g)$ is peaked around its mean value $g_{0}$ with a small standard deviation $\Delta g, g_{\text {eff }}$ is close to $g_{0}$. Then the value of $g / g_{\text {eff }}$ has a small probability to deviate strongly from 1 . Rewriting Eq. (13) in the following form:

$$
\frac{2}{Z} \int_{0}^{\infty} p(g) \frac{\left(1-g / g_{\mathrm{eff}}\right)}{1-(2 / Z)\left(1-g / g_{\mathrm{eff}}\right)} d g=0,
$$

and developing the integrand in power series of $\left(1-g / g_{\text {eff }}\right)$ limited to second order terms, one readily obtains

$$
\frac{g_{0}}{g_{\text {eff }}} \approx 1+\frac{2}{Z}\left(\frac{\Delta g}{g_{0}}\right)^{2} .
$$

When $g_{0}$ and $\Delta g$ are known, this equation can be used to calculate $R_{\text {eff }}=1 / g_{\text {eff. }}$. This result also shows that if $\Delta g / g_{0} \approx$ $10 \%$, then $g_{\text {eff }}$ deviates from $g_{0}$ by less than $1 \%$.

\section{EQUIVALENT BULK CONDUCTIVITY OF A RESISTOR NETWORK}

It is interesting for some applications to approximate a discrete resistor network by a continuous medium. For example, the effective medium approximation for a 3D printed mesh may be used to investigate the electromagnetic properties of the mesh in the long-wavelength limit (wavelength greater than the linear size of the lattice unit cell). In a different but close context, it has been shown that the electromagnetic properties of conductive cellular carbon foams with a cell size of $0.5-1 \mathrm{~mm}$ are well described in a continuous medium approximation up to frequencies around $30 \mathrm{GHz}$ (1 cm wavelength) [38]. Let us consider different ways for the modeling of $3 \mathrm{D}$ printed mesh as a continuous medium.

\section{A. Global definition of the effective conductivity}

The effective conductivity tensor $\sigma_{\text {eff }}$ of an arbitrary inhomogeneous medium is defined by [39-41]

$$
\langle\vec{j}\rangle=\sigma_{\mathrm{eff}}\langle\vec{E}\rangle,
$$

where $\langle\cdots\rangle$ denotes the average of a physical quantity (here the current density $\vec{j}$ or the electric field $\vec{E}$ ) over a characteristic volume of the inhomogeneous medium. In general, $\sigma_{\text {eff }}$ can be determined by solving the electrostatic equation with 
appropriate boundary conditions for the electric potential $\Phi$ $[12,40]$ :

$$
\vec{\nabla} \cdot[\sigma(\vec{r}) \vec{\nabla} \Phi]=0
$$

Interestingly, this equation can be discretized using a finitedifference scheme to yield a set of equations similar to those derived for a discrete resistor network [Eq. (1) with $I_{i}=0$ ].

The effective conductivity $\sigma_{\text {eff }}$ of a periodic resistor network can be addressed as follows. A slice of network, delimited by two parallel planes of specific Miller indices and a distance of $L$ from each other, is contacted by two parallel infinite plates. The nodes of the network in contact with one plate or the other are set to the potentials 0 or $\Phi_{L}$, respectively. These are Dirichlet boundary conditions $[42,43]$. Due to the assumed geometrical periodicity of the lattice, periodic boundary conditions can be imposed on the lattice in directions parallel to the electrodes. The periodic boundary conditions make the sample essentially infinite in two dimensions [12]. They are widely applicable to the modeling of regular or random resistor lattices [12,14,44].

An important advantage of the slab geometry of the problem is that the average electric field is known, and only the average current density $\langle\vec{j}\rangle$ needs to be calculated. From the solution of Eq. (1) with Dirichlet boundary conditions in one direction and periodic boundary conditions in other directions, we can find the electric potential distribution on the inner nodes of the network. Knowing these potentials, we can find the currents $I_{i j}$ flowing from node $i$ to node $j$ through the bond between them:

$$
I_{i j}=g_{i j}\left(V_{i}-V_{j}\right) .
$$

From there the average current density can be obtained as follows. Let $\vec{l}_{i j}$ be the vector in Cartesian space connecting node $i$ to node $j$. Let $\Omega$ be the volume of the network slab that is reproduced periodically in the directions parallel to the electrodes. Then

$$
\langle\vec{j}\rangle=\frac{1}{\Omega} \sum_{\Omega}\left\langle I_{i j} \vec{l}_{i j}\right\rangle,
$$

where the sum is over all the bonds contained in the volume $\Omega$. This relation provides us with the numerical value of the average current density for the imposed averaged electric field $\langle\vec{E}\rangle=\Phi_{L} \vec{n} / L$, where $\vec{n}$ is the unit vector perpendicular to the plate electrodes. The calculations can be performed for electrodes successively set normal to each axis of the Cartesian coordinate system [41]. Using Eq. (16), the nine components of the tensor $\sigma_{\text {eff }}$ can be obtained.

Let us consider the particular case where the lattice is ideal, periodic, and geometrically simple. We imagine a unit cell in the form of a rectangular parallelepiped with resistors along its edges. The lengths of the edges are denoted as $a_{1}, a_{2}$, and $a_{3}$, and the corresponding resistances are $R_{1}, R_{2}$, and $R_{3}$. The unit vectors $\vec{e}_{1}, \vec{e}_{2}$, and $\vec{e}_{3}$ of the Cartesian coordinates are taken parallel to the edges of the unit cell. We take the plate electrodes to be normal to $\vec{e}_{1}$, and the network slab has a thickness $L=N_{1} a_{1}$. The smallest volume $\Omega$ that can be reproduced periodically along $\vec{e}_{2}$ and $\vec{e}_{3}$ contains $N_{1} \times 1 \times 1$ unit cells. Due to the simple shape of the network, all nodes that belong to a same plane parallel to the basis planes have the same electric potential. The current flows only along the
TABLE VI. The effective conductivity $\sigma_{\text {eff }}$ of resistor networks with cubic symmetry in units of $1 /(R a)$ where $R$ is the bond resistance and $a$ is the length of the conventional cubic cell.

\begin{tabular}{lccr}
\hline \hline Diamond & sc & bcc & fcc \\
\hline 1 & 1 & 2 & 2 \\
\hline \hline
\end{tabular}

direction $\vec{e}_{1}$ through linear and parallel chains composed of $N_{1}$ resistances $R_{1}$. Its value is $I_{1}=\Phi_{L} /\left(N_{1} R_{1}\right)$. Equations (16) and (19) readily yield $\sigma_{11}=a_{1} /\left(a_{2} a_{3} R_{1}\right)$. The other diagonal elements of $\sigma_{\text {eff }}$ can be obtained in the same way. The same reasoning is easily applied to lattices with cubic symmetry: diamond, simple cubic (sc), body-centered cubic (bcc), and face-centered cubic (fcc) lattices. Table VI gives the obtained values of the effective conductance, which is diagonal and isotropic.

If the structure of the resistor network is perturbed by defects or by geometrical distortions, the method described above can still be applied by considering a supercell to be reproduced periodically in the lateral directions. Therefore, the computation of $\sigma_{\text {eff }}$ can quite generally be applied to networks with different kinds of inhomogeneities: (1) lattices with nonrandom spatial distribution of defects [45], (2) lattices with fluctuations of the orientation and length of the bonds, and (3) lattices with bond resistances varying in a correlated way.

The very same methodology can be used in practice to characterize $3 \mathrm{D}$ printed meshes. The nodes located on two parallel faces of a mesh are contacted to two electrodes, and the resistance of the whole structure is measured across the electrodes. An effective conductivity is obtained from this measurement. If the lattice is simple like in Table VI, the bond resistance can be deduced. When the lattice has a complex geometric structure and contains different resistors, the same task can be performed, but it may need several measurements on slabs cut along different directions.

\section{B. Local definition of the effective conductivity}

Let us investigate the effective bulk conductivity of a discrete network using a more local point of view. The idea here is to resort to a four-probe-like setup where the current $I_{0}$ is injected at node $i$ and is collected at node $j$, while the potential drop is measured between two other nodes $m$ and $n$. Equation (7) allows us to obtain the apparent resistance $R_{a}=$ $\left(V_{m}-V_{n}\right) / I_{0}$ in a form tractable by the recursion algorithm:

$$
R_{a}=\lim _{y \rightarrow 0+}(\langle u|\mathbf{r}(y)| u\rangle-\langle v|\mathbf{r}(y)| v\rangle),
$$

where

$$
\begin{aligned}
& |u\rangle=\frac{|i\rangle-|j\rangle+|m\rangle-|n\rangle}{2}, \\
& |v\rangle=\frac{|i\rangle-|j\rangle-|m\rangle+|n\rangle}{2} .
\end{aligned}
$$

Equation (20) demands computing two diagonal elements of the resolvent operators $\mathbf{r}(y)$, which is easy to do by the recursion algorithm. 
TABLE VII. Application of Eq. (20) to a simple cubic lattice in the Wenner configuration along the [100] direction, the distance between two successive probes being $l$ times the lattice parameter $a$. The results are given in units of the bond resistance $R$. The last row lists the value of $\rho$ in units of $R a$ and calculated by Eq. (22) from the values of $R_{a}$ contained in the row above.

\begin{tabular}{lllclcl}
\hline \hline$l$ & 1 & 2 & 3 & 4 & 5 & \multicolumn{1}{c}{6} \\
\hline$R_{a}$ & 0.0863 & 0.0452 & 0.0283 & 0.0205 & 0.0160 & 0.0132 \\
$\rho$ & 1.084 & 1.136 & 1.067 & 1.030 & 1.005 & 0.995 \\
\hline \hline
\end{tabular}

The apparent resistance obtained with the very same setup in a continuous and homogeneous $3 \mathrm{D}$ medium is

$$
R_{a}=\frac{\rho}{4 \pi}\left(\frac{1}{d_{m, i}}-\frac{1}{d_{m, j}}-\frac{1}{d_{n, i}}+\frac{1}{d_{n, j}}\right),
$$

where $d_{m, i}$ is the distance between nodes $m$ and $i$ and so on and $\rho$ is the bulk resistivity. In the so-called Wenner configuration [32], the four probes are put along a straight line in the sequence $i, m, n, j$ and are equidistant: $d_{i, m}=d_{m, n}=d_{n, j}=d$. Equation (21) simplifies and yields

$$
\rho=1 / \sigma=4 \pi R_{a} d
$$

If one injects in this relation the apparent resistance computed by Eq. (20) for a resistor network, using four aligned and equidistant nodes, one obtains a local value of the effective resistivity (or the effective conductivity). By definition, it represents the characteristics that a continuous and homogeneous medium should have to produce the same apparent resistance when the measuring electrodes are placed at exactly the same positions as in the discrete network.

In a simple cubic network, let us take the four probes along the [100] direction with interdistance $d=l a, l=1,2 \ldots$, where $a$ is the bond length equal here to the lattice parameter. The results of the computation are given in Table VII, assuming $R=1$ and $a=1$. Here 20 levels of continued fraction were used in a $53 \times 45 \times 45$ supercell. The continued fraction was terminated with the same optimized parameter $s=3.14605$ as used for the equivalent resistances between two nodes. One can deduce from these calculations that the effective resistivity is not a constant, but it converges to the value listed in Table VI when the distance between the electrodes increases. For small values of $l$, there is deviation of $\rho$ from the value of $1 / \sigma_{\text {eff }}$ given by the global approach. This is due to the radial asymmetry of the current injected into the network at node $i$. It can flow only along six equivalent directions in a simple cubic lattice. In a bulk material, the current density is distributed uniformly in all directions. The radial asymmetry of the current flow, which is related to the nonhomogeneous geometry of network, decreases with increasing distance between the current probes $i$ and $j$ and the voltage probes $m$ and $n$. When the distance exceeds several bond lengths, the application of an effective bulk conductivity to a discrete 3D network is justified.

The effective bulk resistivity calculated exactly as for the simple cubic lattice is given in Table VIII for the body-centered cubic structure. Thirty levels of continued fraction were used in a $50 \times 50 \times 50$ supercell with the optimized termination parameter $s=4.177$ (see Sec. IIB). Here two directions are
TABLE VIII. Local bulk resistivity calculated by Eqs. (22) and (20) for the Wenner configuration in the bcc lattice along the [111] and [100] directions. The distance between two successive probes is $l$ multiplied by the first-neighbor distance $\sqrt{3} a / 2$ for [111] direction and $l$ multiplied by the lattice parameter $a$ for [100] direction, respectively. The resistivity $\rho$ is given in units of $R a, R$ is the bond resistance, and $a$ is the cubic lattice parameter.

\begin{tabular}{lcccccc}
\hline \hline Direction & $l$ & 1 & 2 & 3 & 4 & 5 \\
\hline$[111]$ & $\rho$ & 0.550 & 0.535 & 0.513 & 0.505 & 0.501 \\
{$[100]$} & $\rho$ & 0.428 & 0.476 & 0.488 & 0.491 & 0.492 \\
\hline \hline
\end{tabular}

considered to illustrate the anisotropy of the discrete resistor network. The equivalent resistivity seems to converge toward 0.5 (see Table VIII) faster along the [111] than [100] direction.

Obviously the simple electrokinetic equation (17) needs to be corrected if one wants to reproduce the variations of the apparent bulk resistivity of a discrete conducting mesh. Unlike a homogeneous medium, the mesh has an intrinsic length unit, i.e., bond length. Having realized that, a conducting mesh could still be approached by a continuous medium, provided one uses a nonlocal Ohm's law. The formulation of the nonlocal response of the network has to be designed so as to mimic as best as possible the variations of its apparent local resistivity. This question is under investigation.

\section{CONCLUSIONS}

The recursion algorithm initially developed for the quantum mechanical problems has been applied to tasks related to the theory of resistor networks. The recursion method has been demonstrated to be very efficient and accurate for the characterization of the electrical properties of a 2D and 3D finite-size conducting meshes. For accurate consideration of infinite networks by numerical modeling, one needs to use a cluster model with minimized finite-size effect. For this, we propose the core-shell design of the cluster, where the conductance of the resistors in the boundary shell of the cluster is supposed to be increased. Another way to minimize finite-size effects is by adjusting the termination of the continued fraction in the recursion algorithm in order to reproduce the equivalent resistance between two first neighboring nodes. The efficiency of these two approaches has been investigated by the comparison of numerical data and exact analytical results obtained for infinite square and simple cubic networks. The effective-medium theory allows one to model a random resistor network by an ideal resistor networks with effective bond conductance. Numerical calculations, in particular, provided by the recursion method, make it possible to go much beyond this mean-field approach and give access to statistical data. It allows one to analyze the fluctuations of the resistance between two nodes for any particular configuration of current probes. For specific configurations of current probes, it has been shown that the relative dispersion of the equivalent resistance between two nodes is less than the relative dispersion of the resistors themselves. Finally, the question on how to assign a bulk resistivity or bulk conductivity to a $3 \mathrm{D}$ conducting mesh has been addressed. The general definition of the effective conductivity has been applied to 3D conducting meshes. For a slice of the mesh lattice 
between two planar electrodes, an effective bulk conductivity has been defined unambiguously. It reproduces the conductivity of a continuous medium which should have to transport the same average current density from one electrode to another. Following this consideration, the effective conductivity of different resistor networks with cubic symmetry has been calculated. Locally, an apparent resistivity of a conducting mesh is defined in the same way as with a four-probe measurement technique. The apparent resistivity has been calculated for simple cubic and body-centered cubic lattices. The results depend on the crystallographic direction along which the pin electrodes are aligned and on the distance between these electrodes. When the distance between the electrodes exceeds several times the bond length, the apparent resistivity reproduces the resistivity defined globally in a slab, at least for simple lattices.

\section{ACKNOWLEDGMENTS}

This research has benefited from funding from the European Union H2020-MSCA-RISE-2016 Project No. 734164 "Graphene 3D." The authors are thankful to Dr. Konstantin Batrakov and Dr. Polina Kuzhir for fruitful discussions. M.S. acknowledges support from Tomsk State University's Competitiveness Improvement Program.

\section{APPENDIX: CONVERGENCE, COMPUTATIONAL COMPLEXITY, AND ACCURACY OF THE RECURSION ALGORITHM}

In this Appendix, the convergence of the recursion algorithm versus number of continued fraction levels $l$ is examined in the case of a simple cubic lattice of finite size. The first point of interest is how to obtain the equivalent resistance between two first-neighbor sites $i$ and $j$ with a given accuracy. The underlying problem is the solution of the 3D discrete Poisson's equation [Eq. (3)] in the presence of a pair of current source and sink.

The results obtained with the minimal termination parameter $s$ are presented in Fig. 5 versus $l$. Three lattices, composed of $N=10 \times 10 \times 10,20 \times 20 \times 20$, and $50 \times 50 \times 50$ nodes, were considered. For each of them, Fig. 5 allows us to find the number $l(n)$ of continued fraction levels that produces the resistance $R(i, j)$ with a relative error $10^{-n}$. When $n<4, l(n)$ does not depend on the lattice size $N$. For $n>6, l(n)$ increases approximately like the linear size $\sqrt[3]{N}$ of the network.

The number of multiplication operations required by the recursion algorithm is $l(Z+6) N$ with $Z$ the coordination number of the lattice ( $Z=6$ for simple cubic). Then, when the equivalent resistance has to be obtained with more than six exact figures, the number of multiplication operations scales with $N$ like $N^{4 / 3}$. In other words, the computational complexity of the recursion algorithm is $O\left(N^{4 / 3}\right)$.

The second point of interest is linked to the accuracy of the recursion method. The question is how precisely the equivalent resistance $R(i, j)$ between two nodes $i$ and $j$ can be obtained depending on the distance between them. We have addressed that question in an infinite cubic lattice of identical resistors, represented by a cluster of size $N$, either equal to $40 \times 40 \times 40$ or $80 \times 80 \times 80$. The number of continued-fraction levels $l$ for both cases was half the linear size of the cluster, namely, 20 and 40, respectively. The relative positions of the nodes $i$

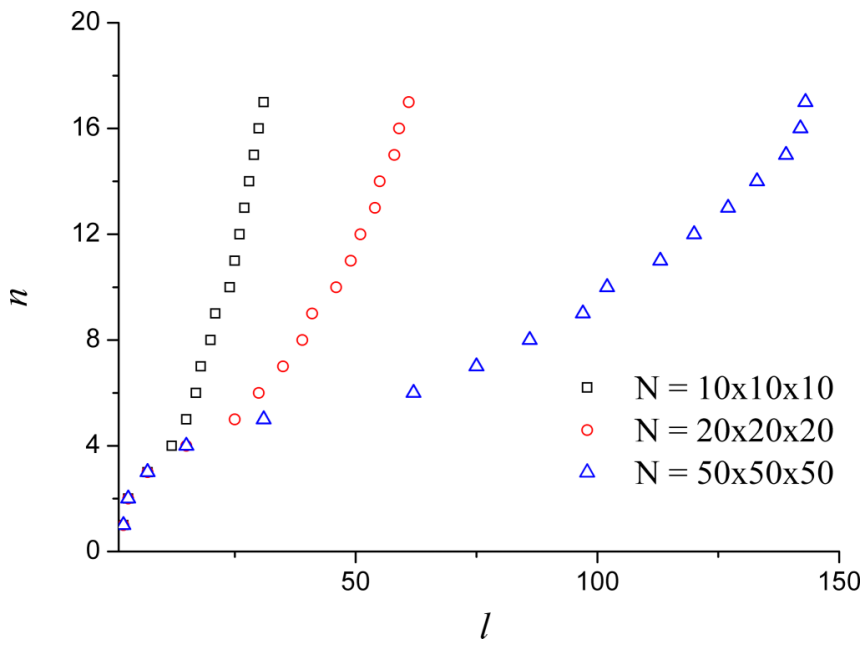

FIG. 5. Relative accuracy of the equivalent resistance $R(i, j)$ between two first-neighbor nodes obtained with a given number $l$ of continued-fraction levels in a simple-cubic lattice of size $N=10 \times$ $10 \times 10,20 \times 20 \times 20$, and $50 \times 50 \times 50$. The relative accuracy is defined by $n=-\log |\delta R(i, j)| / R_{0}$ [see Eq. (9)] with $R_{0}$ the bond rsistance.

and $j$ were taken along three crystallographic directions, as either $(m, 0,0),(m, m, 0)$, or $(m, m, m)$, with $m=1,2, \ldots, 10$. The termination parameter $s$ of the continued fraction (12) was adjusted to reproduce the exact value of the equivalent resistance between two nearest-neighbor nodes $[(1,0,0)$ case]. Setting the bond resistance $R_{0}$ to 1 , the terminations parameters used were $s=3.14605$ for $l=20$ and $s=3.07640$ for $l=40$. The absolute error $\delta R(i, j)$ is plotted in Fig. 6 against $m$ for the three sets of pairs of nodes and for the two cluster sizes. The error increases approximately like $m^{2}$ for each crystallographic direction. For a given $m$, the error increases on going from $(m, 0,0)$ to $(m, m, 0)$ and from $(m, m, 0)$ to $(m, m, m)$. Overall, one can say that $\delta R(i, j)$ increases with the square of the geometrical distance between the nodes $i$ and $j$. For all $m$, the error decreases approximately by a factor of eight by doubling the linear size of the cluster.

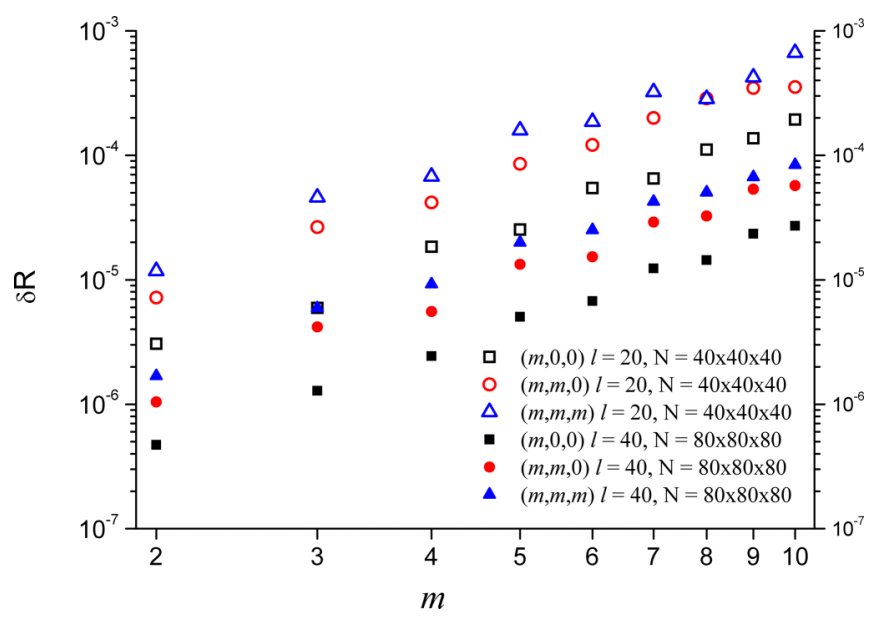

FIG. 6. Plot of the error $\delta R(I, j)$ [Eq. (9)] versus the integer parameter $m$ that sets the distance between the node $i$ at $(0,0,0)$ and the node $j$ at $(m, 0,0),(m, m, 0)$, and $(m, m, m)$. Continued fractions with $l=20$ and 40 levels were used in a cluster of size $2 l \times 2 l \times 2 l$. 
[1] S. S. Babu, L. Love, R. Dehoff, W. Peter, T. R. Watkins, and S. Pannala, MRS Bull. 40, 1154 (2015).

[2] A. Szczurek, A. Ortona, L. Ferrari, E. Rezaei, G. Medjahdi, V. Fierro, D. Bychanok, P. Kuzhir, and A. Celzard, Carbon 88, 70 (2015).

[3] D. Zhang, B. Chi, B. Li, Z. Gao, Y. Du, J. Guo, and J. Wei, Synth. Met. 217, 79 (2016).

[4] G. Postiglione, G. Natale, G. Griffini, M. Levi, and S. Turri, Compos. A: Appl. Sci. Manuf. 76, 110 (2015).

[5] W. Bauhofer and J. Z. Kovacs, Compos. Sci. Technol. 69, 1486 (2009).

[6] J. R. Potts, D. R. Dreyer, C. W. Bielawski, and R. S. Ruoff, Polymer 52, 5 (2011).

[7] Z. Spitalsky, D. Tasis, K. Papagelis, and C. Galiotis, Prog. Polym. Sci. 35, 357 (2010).

[8] E. Fantino, A. Chiappone, I. Roppolo, D. Manfredi, R. Bongiovanni, C. F. Pirri, and F. Calignano, Adv. Mater. 28, 3712 (2016).

[9] M. Letellier, J. Macutkevic, P. Kuzhir, J. Banys, V. Fierro, and A. Celzrad, Carbon 122, 217 (2017).

[10] P. Kuzhir, A. Paddubskaya, D. Bychanok, A. Nemilentsau, M. Shuba, A. Plusch, S. Maksimenko, S. Bellucci, L. Coderoni, F. Micciulla, I. Sacco, G. Rinaldi, J. Macutkevic, D. Seliuta, G. Valusis, and J. Banys, Thin Solid Films 519, 4114 (2011).

[11] N. A. Aal, F. El-Tantawy, A. Al-Hajry, and M. Bououdina, Polym. Compos. 29, 125 (2008).

[12] S. Kirkpatrick, Rev. Mod. Phys. 45, 574 (1973).

[13] J. Bernasconi, Phys. Rev. B 9, 4575 (1974).

[14] J. Koplik, J. Phys. C 14, 4821 (1981).

[15] J. Cserti, Am. J. Phys. 68, 896 (2000).

[16] F. Y. Wu, J. Phys. A 37, 6653 (2004).

[17] M. Othman and A. R. Abdullah, Intl. J. Comp. Math. 76, 203 (2000).

[18] G. S. Joyce, J. Phys. A 5, L65 (1972).

[19] M. Inoue, J. Math. Phys. 16, 809 (1975).

[20] G. S. Joyce, J. Phys. A 31, 5105 (1998).

[21] A. J. Guttmann, J. Phys. A 43, 305205 (2010).

[22] J. H. Asad, A. A. Diab, M. Q. Owaidat, R. S. Hijjawi, and J. M. Khalifeh, Acta Phys. Pol. A 125, 60 (2014).
[23] J. Cserti, G. Szechenyi, and G. David, J. Phys. A 44, 215201 (2011).

[24] J. Cserti, G. David, and A. Piroth, Am. J. Phys. 70, 153 (2002).

[25] M. Q. Owaidat, R. S. Hijjawi, and J. M. Khalifeh, J. Phys. A 43, 375204 (2010).

[26] Y. Notay and A. Napov, J. Comput. Phys. 281, 237 (2015).

[27] D. G. Pettifor and D. L. Weaire, The Recursion Method and Its Applications (Springer Verlag, Berlin, 1985).

[28] M. A. Jafarizadeh, R. Sufiani, and S. Jafarizadeh, J. Phys. A 40, 4949 (2007).

[29] R. Haydock, V. Heine, and M. J. Kelly, J. Phys. C 5, 2845 (1972).

[30] F. W. Dorr, SIAM Rev. 12, 248 (1970).

[31] R. Chandra, Ph.D. thesis, Yale University, 1978.

[32] I. Miccoli, F. Edler, H. Pfnür, and C. Tegenkamp, J. Phys.: Condens. Matter 27, 223201 (2015).

[33] D. J. Frank and C. J. Lobb, Phys. Rev. B 37, 302 (1988).

[34] R. J. LeVeque, Finite difference methods for differential equations, lecture notes, University of Washington, Seattle (unpublished).

[35] P. M. Osterberg and A. S. Inan, Am. J. Phys. 72, 972 (2004).

[36] S. Giordano, Intl. J. Circuit Theory Appl. 33, 519 (2005).

[37] C. O'Callaghan, C. Gomes da Rocha, H. G. Manning, J. J. Boland, and M. S. Ferreira, Phys. Chem. Chem. Phys. 18, 27564 (2016).

[38] D. Bychanok, A. Plyushch, K. Piasotski, A. Paddubskaya, S. Voronovich, P. Kuzhir, S. Baturkin, A. Klochkov, E. Korovin, M. Letellier, S. Schaefer, A. Szczurek, V. Fierro, and A. Celzard, Phys. Scr. 90, 094019 (2015).

[39] D. Stroud, Phys. Rev. B 12, 3368 (1975).

[40] J. Helsing and A. Helte, J. Appl. Phys. 69, 3583 (1991).

[41] Y. M. Strelniker and D. J. Bergman, Phys. Rev. B 50, 14001 (1994).

[42] Y. Hakobyan, K. D. Papoulia, and M. D. Grigoriu, Phys. Rev. B 76, 144205 (2007).

[43] N. Athanasopoulos and V. Kostopoulos, Compos. Sci. Technol. 72, 1273 (2012).

[44] S. Roux and H. J. Herrmann, Europhys. Lett. 4, 1227 (1987).

[45] J. L. Duckers and R. G. Ross, Phys. Lett. A 49, 361 (1974). 